\title{
RUMOURS WITH GENERAL INITIAL CONDITIONS
}

\author{
SELMA BELEN ${ }^{1}$ and C. E. M. PEARCE ${ }^{1}$
}

(Received 24 January, 2002; revised 5 November, 2003)

\begin{abstract}
An analysis is made of the Daley-Kendall and Maki-Thompson rumour models starting from general initial proportions of ignorants, spreaders and stiflers in the population. We investigate as a function of the initial conditions the composition of the final population when the rumour has run its course.
\end{abstract}

\section{Introduction}

The stochastic theory of rumours, with interacting subpopulations of ignorants, spreaders and stiflers, began with the seminal paper of Daley and Kendall [4]. The most striking result in the area-that if there is one spreader initially, then the proportion of the population never to hear the rumour converges almost surely to a proportion 0.203188 of the population size as the latter tends to infinity-was first signalled in that article. This result occurs also in the variant stochastic model of Maki and Thompson [5], although a typographic error has resulted in the value 0.238 being cited in a number of consequent papers.

A rigorous treatment of the limiting behaviour of these processes proved unexpectedly tricky and the literature has mainly addressed technical questions of stochastic convergence, mostly via diffusion-type approximations and martingale arguments (see, for example, Barbour [1], Sudbury [7], Pittel [6] and Watson [8]). Broader questions for those models are still largely unexplored. For example, the standard assumption that a rumour is initiated by a single spreader, while doubtless true in many concrete examples, is certainly inappropriate for others in this current age of mass communication, where a rumour may be initiated by television or radio.

In this paper we adopt general initial conditions and consider the evolution of the models. We examine how the initial conditions bear on what proportion of ignorants

\footnotetext{
${ }^{1}$ School of Applied Mathematics, University of Adelaide, Adelaide SA 5005, Australia; c-mail: cpearce@maths.adelaide.edu.au.
}

(C) Australian Mathematical Society 2004, Serial-fee code 1446-1811/04 
and of the total population never get to hear the rumour. Our formulæ have direct consequences to several questions of physical interest which we shall treat fully elsewhere.

We discover inter alia the perhaps surprising result that, even when the initial proportion of spreaders in the population tends to unity, the fraction of the subpopulation of initial ignorants that never hear the rumour does not approach zero.

In order to uncover some new results without becoming enmeshed in technicalities we shall adopt a broad brush stroke and, after introducing stochastic rumours in Section 2, proceed using a continuous deterministic approximation via differential equations as in the seminal article of Daley and Kendall [4]. In Section 3 we treat the evolution of the deterministic model with time. In Section 4 we find the proportion of the initial ignorants who never hear the rumour and in Section 5 the proportion of the whole population who never hear the rumour.

\section{The model}

A stochastic rumour pertains to a fixed population of $n$ individuals consisting of subpopulations of ignorants, spreaders and stiflers. Homogeneous mixing of individuals occurs, with a given proportion of ignorant-spreader interactions leading to the ignorant becoming a spreader and the same proportion of spreader-stifler interactions resulting in the spreader becoming a stifler. A similar phenomenon occurs with spreader-spreader interactions. In the Daley-Kendall version, the outcome is two stiflers. The Maki-Thompson version distinguishes between an initiating and a receptor spreader in such an interaction and only one spreader converts to being a stifler as a result of the encounter.

With these models a sequence of state transitions occurs. These are of three types. Let $i, s, r$ be the respective numbers of ignorants, spreaders and stiflers at a given moment. The ignorant-spreader and spreader-stifler interactions respectively may be expressed as state transitions

$$
\begin{aligned}
& (i, s, r) \longrightarrow(i-1, s+1, r) \\
& (i, s, r) \longrightarrow(i, s-1, r+1)
\end{aligned}
$$

The spreader-spreader interaction is

$$
(i, s, r) \longrightarrow(i, s-2, r+2)
$$

for the Daley-Kendall version of the process and

$$
(i, s, r) \longrightarrow(i, s-1, r+1)
$$


for the Maki-Thompson version. We remark that (2.1) and (2.2) are formally the same, though the first (the spreader-stifler interaction) occurs at a rate proportional to $s r$ and the latter (the spreader-spreader interaction) at a rate proportional to $s(s-1)$. Such sequences lead inexorably (after a finite number of transitions) to states in which there are no spreaders left.

We restrict our attention to the limiting form of these models as the total population size tends to infinity. We adopt a continuum formulation. Let $i(t), s(t), r(t)$ denote respectively the proportions of the total population at time $t$ that are respectively ignorants, spreaders and stiflers. With an appropriate choice of time scale, the common coefficient for interactions leading to a change of subpopulation of an individual can be taken as unity. The Daley-Kendall and Maki-Thompson stochastic models lead to the same set of coupled deterministic subpopulation equations

$$
\begin{aligned}
d i / d t & =-i s, \\
d s / d t & =-s(1-2 i), \\
d r / d t & =s(1-i),
\end{aligned}
$$

which apply in the limit of a total population size tending to infinity.

We adopt the initial conditions

$$
i(0)=\alpha>0, \quad s(0)=\beta>0, \quad r(0)=\gamma, \quad \text { with } \quad \alpha+\beta+\gamma=1 .
$$

We remark that (2.5) may be considered redundant, since

$$
r=1-i-s .
$$

It is convenient to introduce the parameter $\theta=\theta(\tau):=i / \alpha$, the ratio of the proportion of ignorants at time $\tau$ to the initial proportion. In the next section we address the dynamics and asymptotics of the continuum rumour process.

\section{Evolution of the system}

THEOREM 1. The evolution of the rumour process prescribed by (2.3)-(2.6) is given parametrically in terms of $i$ by

$$
\begin{aligned}
s & =\beta-2(i-\alpha)+\ln (i / \alpha) \\
& =\beta-2 \alpha(\theta-1)+\ln \theta
\end{aligned}
$$

and (2.7).

The process evolves towards an asymptotic state $\left(i_{\infty}, 0, r_{\infty}\right)$, with

$$
i \downarrow i_{\infty}=i_{\infty}(\alpha, \beta) \text { as } \tau \rightarrow \infty
$$


and

$$
0<i_{\infty}<1 / 2
$$

The parameter $\theta_{\infty}:=i_{\infty} / \alpha$ satisfies the transcendental equation

$$
\theta_{\infty} e^{2 \alpha\left(1-\theta_{\infty}\right)}=e^{-\beta}
$$

Further, $s \rightarrow 0$ and $r(\tau) \uparrow r_{\infty}=1-i_{\infty}$ as $\tau \rightarrow \infty$.

ProOF. Equation (2.3) implies that $i$ is a strictly decreasing function of $\tau$ and may therefore be used as an independent parameter. Combining (2.3) and (2.4) provides the relation

$$
\frac{d s}{d i}=\frac{1-2 i}{i},
$$

which integrates to give (3.1). The value of $r$ is then determined by (2.7).

Being strictly decreasing and bounded below by zero and above by unity, $i$ must therefore tend to some limit $i_{\infty}<1$ as $\tau \rightarrow \infty$. By (2.5), $r$ is strictly increasing with time. Since it is bounded above by unity, it must tend to a limit $r_{\infty}>0$ as $\tau \rightarrow \infty$. Also, since $i_{\infty}<1$, we have from (2.5) that $s \rightarrow 0$ as $\tau \rightarrow \infty$, or equivalently $s \rightarrow 0$ as $i \rightarrow i_{\infty}$.

If $\alpha \leq 1 / 2$, then $i(\tau)<1 / 2$ for all $\tau>0$ since $i$ is strictly decreasing, and hence $i_{\infty}<1 / 2$. If $\alpha>1 / 2$, then by (3.5) $d s / d i<0$ and

$$
\frac{d s}{d \tau}=\frac{d s}{d i} \cdot \frac{d i}{d \tau}>0
$$

initially. Since $s \rightarrow 0$ as $\tau \rightarrow \infty, s(\tau)$ must first increase to a local (and global) maximum (at which time $i=1 / 2$ ) and by (3.5) decrease thereafter. Because $i$ is strictly decreasing, we thus have $i_{\infty}<1 / 2$. Since $r_{\infty}>0$, we have also that $i_{\infty}=1-r_{\infty}>0$.

Finally, letting $\tau \rightarrow \infty$ in (3.2) yields

$$
0=\beta-2 \alpha\left(\theta_{\infty}-1\right)+\ln \theta_{\infty}
$$

which is just (3.4).

Equation (3.6) may be expressed as

$$
w e^{w}=-2 \alpha e^{-2 \alpha-\beta}
$$

where $w:=-2 \alpha \theta_{\infty}$. 


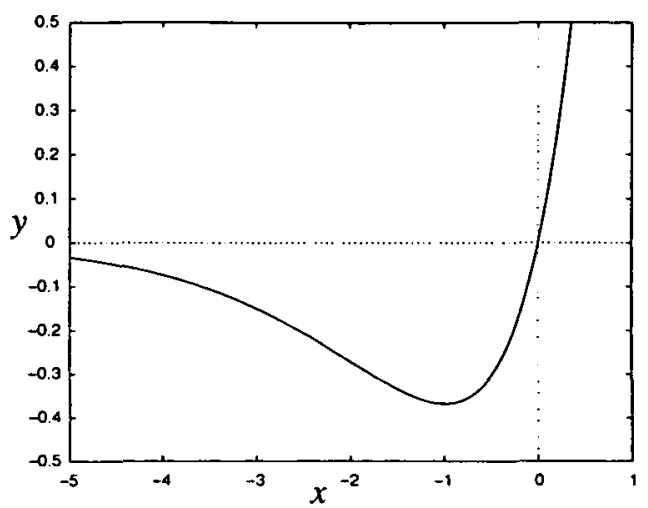

FIGURE 1. The graph of the equation $y=x e^{x}$

The equation

$$
x e^{x}=y
$$

has two real solutions when $-1 / e<y<0$ (see Figure 1). For $\alpha, \beta>0$ we have $-2 \alpha e^{-2 \alpha}<-2 \alpha e^{-2 \alpha-\beta}$, so that $\left.x e^{x}\right|_{x=-2 \alpha}<\left.x e^{x}\right|_{x=-2 \alpha \theta}$ for $0<\alpha<1$. Hence one of the real solutions of (3.7) is less than $-2 \alpha$ and the other greater than $-2 \alpha$. As we must have $0<\theta<1$, the physical solution to (3.7) is the one greater than $-2 \alpha$, that is, the numerically smaller real solution of (3.8). The function $w=w(y)$ giving the unique real solution to (3.8) for $y \geq 0$ and the numerically smaller real solution for $y<0$ has been in the literature for over 200 years and is known as the Lambert $W$ function (see [2]). Lagrange's expansion provides an explicit series evaluation

$$
w=\sum_{k=1}^{\infty} \frac{(-y)^{k}}{k !} k^{k-1}
$$

Thus for $\alpha, \beta>0$

$$
\theta=-\frac{1}{2 \alpha} w\left(-2 \alpha e^{-2 \alpha-\beta}\right)=\sum_{k=1}^{\infty} \frac{(-2 \alpha k)^{k-1}}{k !} \exp (-k(2 \alpha+\beta)) .
$$

\section{Proportion of ignorants never hearing the rumour}

THEOREM 2. (a) For $\alpha+\gamma=1-\beta$ fixed, $\theta_{\infty}$ is strictly decreasing in $\alpha$.

(b) For $\beta+\gamma=1-\alpha$ fixed, $\theta_{\infty}$ is strictly decreasing in $\beta$.

(c) For $\alpha+\beta=1-\gamma$ fuxed, $\theta_{\infty}$ is strictly decreasing in $\alpha$. 
PROOF. Consider situation (a). Implicit differentiation of (3.6) with respect to $\alpha$ provides

$$
\frac{\partial \theta_{\infty}}{\partial \alpha}=-\frac{\theta_{\infty}\left(1-\theta_{\infty}\right)}{1-2 \alpha \theta_{\infty}},
$$

which is negative by (3.3), since $\alpha \theta_{\infty}=i_{\infty}$. This establishes the result in (a).

Similarly in the context of (b), we have

$$
\frac{\partial \theta_{\infty}}{\partial \beta}=-\frac{\theta_{\infty}}{1-2 \alpha \theta_{\infty}}<0,
$$

giving the requisite result.

Finally, for (c), suppose $\alpha+\beta=c$, fixed, so that $\theta_{\infty}=\theta_{\infty}(\alpha)$ and

$$
c-\alpha-2 \alpha\left(\theta_{\infty}-1\right)+\ln \theta_{\infty}=0 .
$$

Implicit differentiation yields

$$
\frac{d \theta_{\infty}}{d \alpha}=-\frac{2 \theta_{\infty}\left(1-\theta_{\infty}\right)}{1-2 \alpha \theta_{\infty}}<0,
$$

and we are done.

Figure 2 depicts the situation for case (c) with the standard $\gamma=0$, so that $\alpha+\beta=1$. For simplicity, we commit an abuse of notation and set $\theta(\alpha)=\theta_{\infty}(\alpha)$. We have seen that $\theta$ is a strictly monotone decreasing function of $\alpha$ on $(0,1)$. Its infimum satisfies the Daley-Kendall equation $2(1-\theta)+\ln \theta=0$ and is $\theta(1) \approx 0.2031878$. The other real solution $\theta=1$ to this equation is aphysical, as noted in [4]. The supremum of $\theta$ is $\theta(0)=1 / e \approx 0.36787944$. That is, we have the somewhat surprising result that when nearly all the population are initially spreaders, it is still the case that a proportion $1 / e$ of the initial ignorants never hear the rumour.

The infimum value $\theta(1)$ arises in the limit of total population tending to infinity for a fixed finite initial number of spreaders. The supremum value $\theta(0)$ arises similarly with a fixed finite number of ignorants.

Despite the suggestion from Figure 2, $\theta$ is not a concave function of $\alpha$ throughout $(0,1)$. We may see this as follows. Implicit differentiation of (3.6) twice with respect to $\alpha$ yields

$$
\theta(1-2 \alpha \theta) \frac{d^{2} \theta}{d \alpha^{2}}=\frac{d \theta}{d \alpha}\left[4 \theta^{2}+\frac{d \theta}{d \alpha}\right]
$$

or, using (4.1),

$$
(1-2 \alpha \theta) \frac{d^{2} \theta}{d \alpha^{2}}=\frac{d \theta}{d \alpha}\left[4 \theta-\frac{1-2 \theta}{1-2 \theta \alpha}\right]
$$




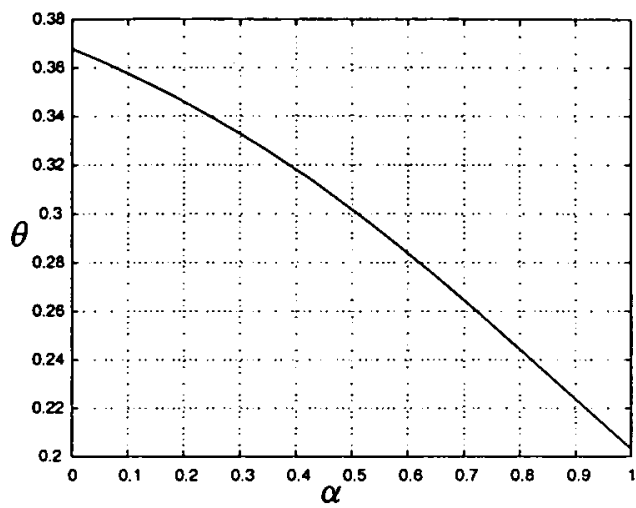

FIGURE 2. The behaviour of the function $\theta$

For $\alpha \approx 0$, the expression in brackets on the right is $\approx 4 \theta(0)-[1-2 \theta(0)]>0$, so $d^{2} \theta / d \alpha^{2}$ is negative and $\theta$ is a strictly concave function of $\alpha$. On the other hand, for $\alpha \approx 1$, the expression in brackets is $\approx 4 \theta(1)-1<0$, so $d^{2} \theta / d \alpha^{2}$ is positive and $\theta$ is a strictly convex function of $\alpha$.

In the concluding section we examine the variation of $\zeta:=i_{\infty}=\alpha \theta_{\infty}$ with the initial conditions.

\section{Proportion of total population never hearing the rumour}

The dependence on initial conditions of the proportion $\zeta$ of the total population who never hear the rumour is also of interest.

THEOREM 3. (a) For $\alpha+\gamma=1-\beta$ fixed, $\zeta$ is strictly increasing in $\alpha$ for $\alpha<1 / 2$ and strictly decreasing in $\alpha$ for $\alpha>1 / 2$.

(b) For $\beta+\gamma=1-\alpha$ fixed, $\zeta$ is strictly decreasing in $\beta$.

(c) For $\alpha+\beta=1-\gamma$ fuxed, $\zeta$ is strictly increasing in $\alpha$.

Proof. We may rewrite (3.6) as

$$
\beta-2(\zeta-\alpha)+\ln \zeta-\ln \alpha=0
$$

The argument now follows that of Theorem 2. In (a), (b), (c) we have respectively from implicit differentiation of (5.1) that

$$
\frac{\partial \zeta}{\partial \alpha}=\frac{\zeta}{\alpha} \cdot \frac{1-2 \alpha}{1-2 \zeta}, \quad \frac{\partial \zeta}{\partial \beta}=\frac{-\zeta}{1-2 \zeta}, \quad \frac{d \zeta}{d \alpha}=\frac{\zeta}{\alpha} \cdot \frac{1-\alpha}{1-2 \zeta},
$$

from which the conclusions follow directly, since $\zeta<1 / 2$. 
COROLlary 4. We have $\zeta^{*}:=\sup \zeta=1 / 2$. This occurs in the limiting case $\alpha=1 / 2=\gamma$, with $\beta=0$.

PROOF. From (c) of Theorem 2, we have for fixed $\gamma$ that $\zeta$ has supremum approached in the limit $\alpha=1-\gamma$ with $\beta=0$. But from (a), we have in the limit $\beta=0$ that $\zeta$ has supremum arising from $\alpha=1 / 2$. This gives the second part of the enunciation.

From $(5.1), \zeta^{*}$ satisfies

$$
0=-2 \zeta^{*}+1+\ln \left(2 \zeta^{*}\right)
$$

For $x>0$, set $g(x):=\ln x-x+1$. Then $g$ is strictly increasing on $(0,1)$ and strictly decreasing on $(1, \infty)$. It follows that $x=1$ is the only solution to $g(x)=0$. We deduce from (5.2) that $\zeta^{*}=1 / 2$.

\section{References}

[1] A. D. Barbour, "The principle of the diffusion of arbitrary constants", J. Appl. Prob. 9 (1972) 519-541.

[2] R. M. Corless, G. H. Gonnet, D. E. G. Hare, D. J. Jeffrey and D. E. Knuth, "On the Lambert W function.", Adv. Comp. Math. 5 (1996) 329-359.

[3] D. J. Daley and J. Gani, Epidemic modelling (Cambridge University Press, UK, 1999).

[4] D. J. Daley and D. G. Kendall, "Stochastic rumours", J. Inst. Math. Appl. 1 (1965) 42-55.

[5] D. P. Maki and M. Thompson, Mathematical models and applications (Prentice-Hall, Englewood Cliffs, 1973).

[6] B. Pittel, "On a Daley-Kendall model of random rumours", J. Appl. Prob. 27 (1990) 14-27.

[7] A. Sudbury, "The proportion of the population never hearing a rumour", J. Appl. Prob. 22 (1985) 443-446.

[8] R. Watson, “On the size of a rumour”, Stochastic Process. Appl. 27 (1987) 141-149. 